\title{
Intubating a Treacher Collins Syndrome patient with STRIVE Hi and videolaryngoscopy
}

\author{
Lachlan Young ${ }^{1}$, De Wet Potgieter ${ }^{1}$, Courtney Roche ${ }^{1}$
}

\section{Abstract}

Treacher Collins Syndrome (TCS) is characterised by craniofacial abnormalities which pose a unique airway challenge for anaesthetists. For these patients requiring general anaesthesia and endotracheal intubation, maintaining spontaneous breathing throughout induction should be considered foundational to safety. Spontaneous respiration using intravenous anaesthesia and high-flow nasal oxygen (STRIVE Hi) has been previously shown to safely induce patients with difficult airways. This case demonstrates the first described successful intubation of an adult TCS patient with hyperangulated blade videolaryngoscopy "GlideScope LoPro S4" using a STRIVE Hi induction technique.

Key Words: Treacher Collins Syndrome intubation; STRIVE Hi; Videolaryngoscopy

Introduction

Treacher Collins Syndrome is a disorder of the first and second branchial arches in neural crest formation [1]. It is caused by a mutation on chromosome 5 and has an incidence of 1 in 40000-70000 births [2]. The syndrome is characterised by symmetrical hypoplasia of the maxilla, mandible, and zygomatic process [3]. These individuals are likely to present with a small oral aperture, abnormalities of the temporomandibular joint, retrognathia and palate deformities [3].

TCS is associated with difficult intubation, which has been demonstrated within a paediatric cohort to increase with age $[4,5]$. Current literature does not contain a comprehensive review of airway difficulty and management amongst an adult TCS population; this may represent the contemporary availability for corrective facial surgery [6]. From the outset we suggest caution is applied to generalising paediatric reviews towards adult patients, as airway features in syndromic patients inherently change with age [7].

Previous case studies have demonstrated difficulty with intubation and found success in second generation laryngeal mask airways

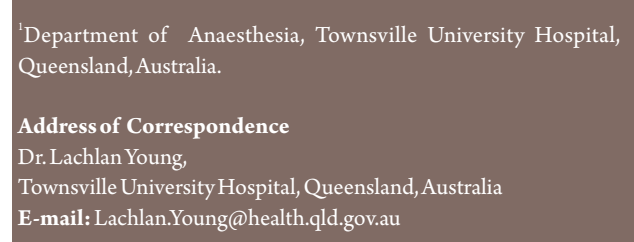

(LMA) amongst adult patients [8,9]. Paloma [8] used an LMA-Fastrach for an emergent caesarean section with no reported complications. Within this study blind intubation through the LMA was attempted, however was unsuccessful. An i-gel was successfully used as backup for Soh [9] after two failed videolaryngoscopies and two failed trans-nasal fibreoptic intubations attempts for a 25-year-old male presenting for emergencybowel resection.

STRIVE Hi focuses on maintaining spontaneous ventilation and utilising the advantages of high-flow nasal oxygen (HFNO) during general anaesthesia. HFNO has gained traction within anaesthesia with physiological benefits for spontaneously breathing patients including generation of positive airway pressure, increased $\mathrm{FiO} 2$, decreased ventilatory dead space and reduced upper airway resistance [10-12]. Propofol has been the favoured anaesthetic agent throughout the history of STRIVE Hi for its titratability and ability to suppress airway reflexes [13]. Most recently it has demonstrated safety in elective structurally obstructed airway surgery [14] and emergency epiglottitis intubation [15].

This case presents the first described successful intubation of an adult TCS patient using a STRIVE Hi induction protocol previously described [14]. Written consent was obtained from the patient to write this case study, and for clinical photography, prior to submission of the manuscript.

\section{Case Report}

A 76-year-old gentleman with Treacher Collins Syndrome was transferred to Townsville Hospital (TTH) for surgical management of choledocolithiasis. He previously presented to a regional hospital with epigastric pain and an obstructive liver function test profile. CT scanning identified an obstructing $27 \mathrm{~mm}$ suspected stone with $18.5 \mathrm{~mm}$ of proximal common bile duct dilatation. He remained haemodynamically stable and afebrile throughout the period of diagnosis and transfer. His past medical history contained hypertension and gastroesophageal reflux disease, for which he was effectively medically treated with amlodipine, perindopril, and esomeprazole. The patient had previously been provided a general anaesthetic more than twenty years ago for a hernia repair, however no documentation surrounding this could be sourced. He lived alone, was completely independent with tasks of daily living and maintained a comfortable exercise tolerance of greater than four metabolic equivalents. $\mathrm{He}$ abstained from both alcohol and tobacco. Upon arrival to the ward at TTH the patient was promptly referred to anaesthetics for operative planning given his anticipated difficult airway. Upon review the patient was noted to have significant craniofacial dysmorphia: facial asymmetry, retrognathia,

(C) 2020 by Journal of Anaesthesia and Critical Care Case Reports|Available on www.jaccr.com | DOI: 10.13107/jaccr.2020.v06i02.147 This is an Open Access article distributed under the terms of the Creative Commons Attribution Non-Commercial License (http://creativecommons.org/licenses/by-nc/3.0) which permits unrestricted non-commercial use, distribution, and reproduction in any medium, provided the original work is properly cited. 


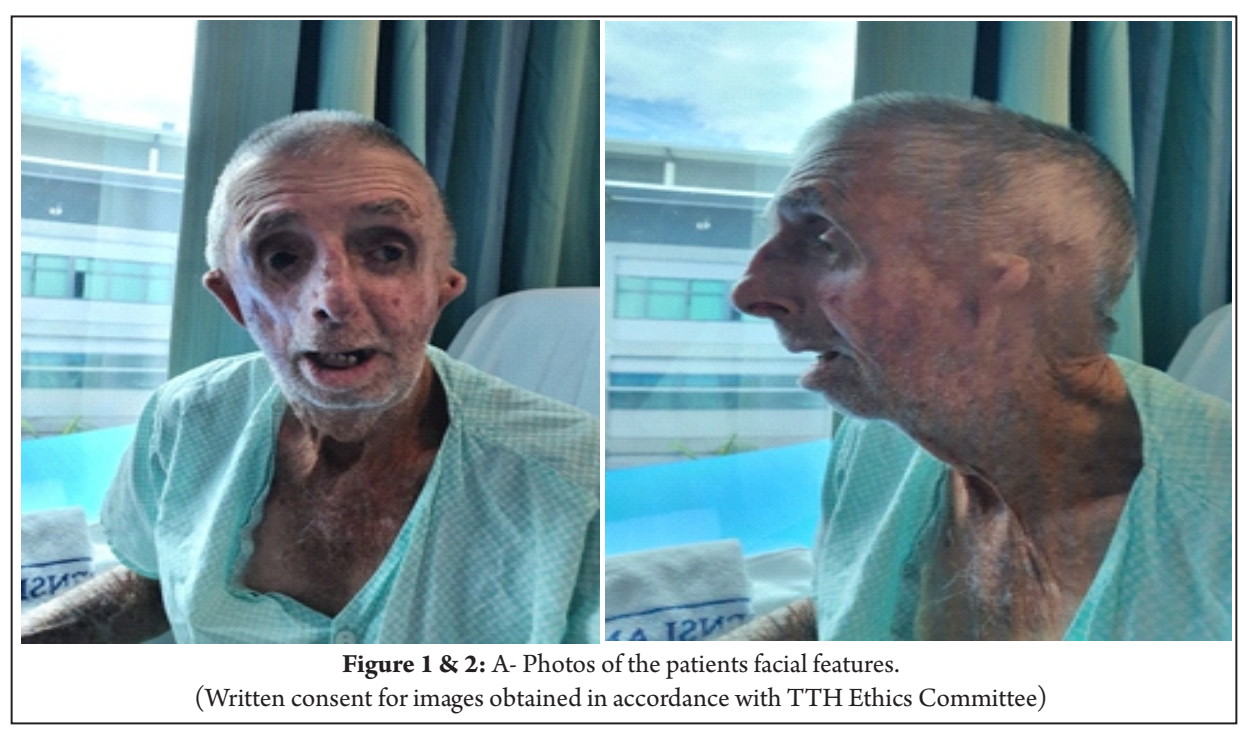

micrognathia, nasal bridge deformity and teeth agenesis (Figures 1 and 2). Bilateral ear malformations were present which conferred a prominent lifelong conductive hearing deficit. On examination his mouth opening was limited to two centimeters, thyromental distance was less than four centimeters and his range of neck motion was poor. Examination identified no stigmata of systemic respiratory or cardiovascular disease. This gentleman was consented for an open cholecystectomy and bile duct exploration to take place during daytime hours with a senior anaesthetist and anaesthetic fellow rostered to the list.

As this procedure represented major abdominal surgery with variable operating time, an endotracheal tube (ETT) was the optimal airway. Given the prospected difficulty of airway management, STRIVE Hi induction was chosen as the induction technique using a protocol previously

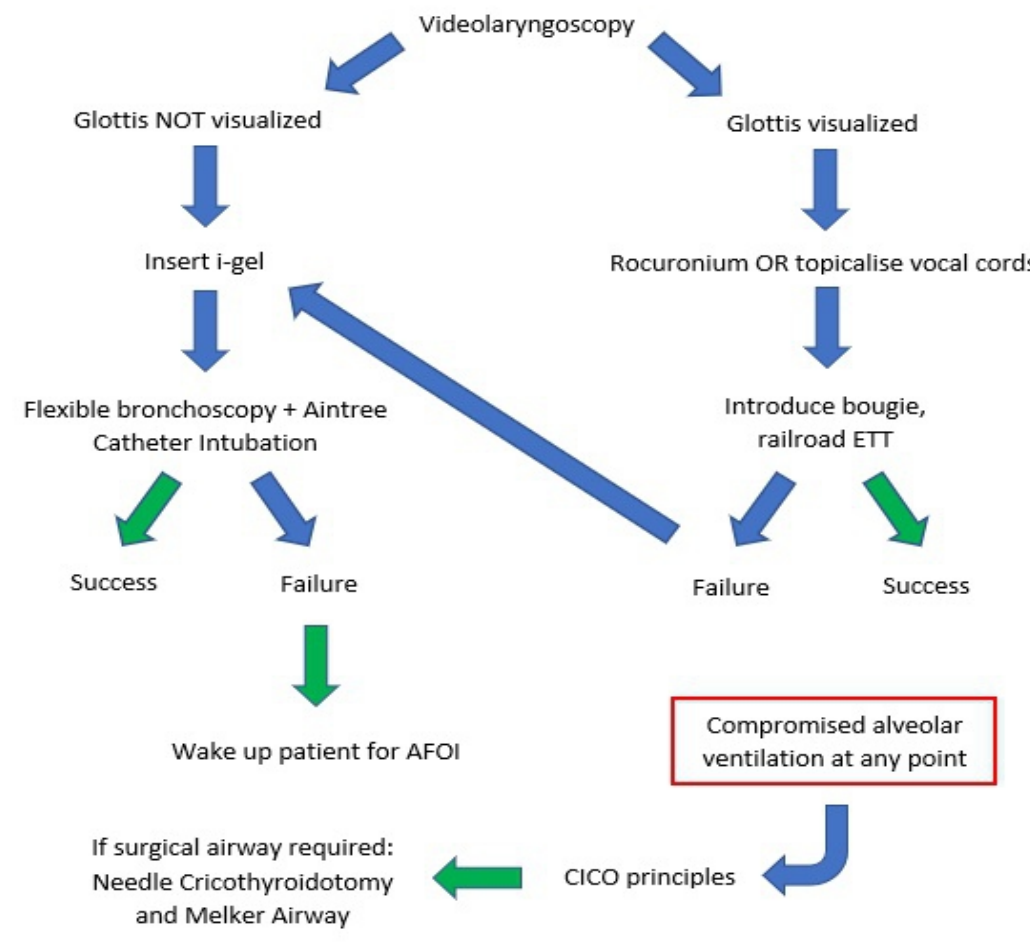

Figure 3: Airway plan following the possibilities of videolaryngoscopy. Videolaryngoscopy "GlideScope LoPro S4" (Verathon, Washington, USA); Intubating catheter "Aintree Catheter" (Cook Medical, Bloomington, Indiana); Second generation LMA "i-gel" (Intersurgical Ltd., Wokingham, UK); Cricothyroidotomy catheter "Melker Airway" (Cook Medical, Bloomington, Indiana) AFOI = 'Awake Fibre Optic Intubation'; BVM = 'Bag Valve Mask'; CICO = 'Cant Intubate Can’t Oxygenate'.

described [14]. The patient was consulted preoperatively by the lead anaesthetist who gained verbal consent and developed an airway plan shown in (Figure 3). The patient strongly favoured the option of STRIVE Hi over awake fibre optic intubation (AFOI).

Preoperatively a radial arterial line and two intravenous cannulae were inserted. HFNO (Optiflow THRIVE; Fisher and Paykel Healthcare) was introduced to the patient at $15 \mathrm{~L} / \mathrm{min}$ and titrated over three minutes to $50 \mathrm{~L} / \mathrm{min}$, which was well tolerated. Glycopyrrolate $400 \mathrm{mcg}$ was provided intravenously as an antisialagogue and CoPhenylcaine was administered to the oropharynx. Induction began with a propofol target-controlled infusion (TCI) using the Marsh-model at a plasma site ( $\mathrm{Cp})$ of 2 $\mathrm{ug} / \mathrm{mL}$. Once the difference between $\mathrm{Cp}$ and effect site $(\mathrm{Ce})$ was less than or equal to 1 $\mathrm{ug} / \mathrm{mL}$, the $\mathrm{Cp}$ was incrementally increased by $1 \mathrm{ug} / \mathrm{mL}$. The patient lost consciousness at Ce $3 \mathrm{ug} / \mathrm{mL}$. At this time, the HFNO flow was increased to $70 \mathrm{~L} / \mathrm{min}$. Stepwise increase in Cp stopped at $6 \mathrm{ug} / \mathrm{mL}$ and Ce was given time to equilibrate. The patient maintained spontaneous respiration throughout this process with no requirement for airway adjuncts. A GlideScope LoPro S4 laryngoscope (Verathon, Washington, USA) was gently introduced by the anaesthetic fellow and identified a complete glottic view, with percentage of glottic opening (POGO) $100 \%$. Lignocaine $1 \%$ was applied directly to the vocal cords and propofol $\mathrm{Cp}$ increased to $8 \mathrm{ug} / \mathrm{mL}$. The laryngoscope was removed and HFNO flow continued at $70 \mathrm{~L} / \mathrm{min}$. After waiting 30 seconds the laryngoscope was reintroduced, vocal cord view was reidentified, and a hyperangulated bougie was passed between the cords. A size 8.0 ETT was railroaded into the trachea- position was confirmed with capnography and auscultation. Throughout induction and intubation oxygen haemoglobin saturations did not fall below $98 \%$, end tidal CO2 did not increase above $45 \mathrm{mmhg}$ and haemodynamic stablility was preserved with small aliquots of metaraminol ( $2 \mathrm{mg}$ total). Remifentanil then accompanied propofol to complete the total intravenous anaesthetic (TIVA). Cp was decreased to $4 \mathrm{ug} / \mathrm{ml}$ for maintenance of anaesthesia. The operation proceeded with no complication and extubation was uneventful. The patient was discharged at day three postoperatively. 


\section{Discussion}

In a previous case study of unsuccessful intubation, authors discussed a narrow mouth opening (1.5 finger breadths) and small supraglottic area as the major contributing anatomical features to this difficult intubation [9]. Similar facial features were present for our patient, who had a small oral aperature and profound retrognathia, which were considered and influenced the decision to use a GlideScope LoPro S4 laryngoscope. GlideScope Spectrum Singleuse are a range of videolaryngoscopes which include varying sizes of the hyperangulated low profile "LoPro" and Macintosh "DirectView MAC" styles [16]. The LoPro in contrast to the MAC exaggerates the angulation of the blade providing the best video view of the vocal cords achievable. The disadvantage of this style is reliance on video view, comparative difficulties with introducing the bougie, and occasional resistance passing the ETT around the axis of the oropharynx. Because of the degree of retrognathia and previously described difficulties by Soh [9] with a MAC blade, a LoPro S4 was considered to offer the best first videolaryngoscopic attempt.

Fibreoptic trans-nasal intubation and intubation through an LMA have previously been successful amongst paediatric patients and were considered as secondary plans to the method utilised [4]. Fibreoptic nasal intubation was potentially difficult due to an unknown degree of maxillary dysmorphism whilst videolaryngscopy offered equipment familiarity and simplicity. Supported by success in previous case studies $[8,9]$ and following the Vortex algorithm [17], an i-gel was readily available in the event of airway compromise. Personal experience of the lead anaesthetist with a background as an intensivist influenced the choice of needle cricothyoidotomy and insertion of a Melker Airway as the CICO surgical airway option. Booth [14] demonstrated success with STRIVE Hi among 30 patients presenting for elective tubeless surgery upon severely obstructed airways. Specific to the induction of anaesthesia, there were no episodes in this cohort of oxygen desaturation below 97\% and no period of airway obstruction or apnoea. More recently STRIVE Hi was extended to safely intubate an adult patient with acute epiglottitis [15]. This featured a 54-year-old male who was stridorous with laboured breathing and impending airway collapse, who was successfully induced with a STRIVE Hi technique and intubated with a C-MAC videolaryngoscope (Karl Storz, Tuttlingen, Germany), bougie and size 6 endotracheal tube. Lee [15] discussed the importance of familiarity with the process avoiding apnoea whilst achieving consistent end points for airway instrumentation. We strongly support this statement; that an anaesthetist must be well versed in the process of STRIVE Hi in elective surgery settings, before extending it into airway emergencies or suspected difficult intubations. The safety of STRIVE Hi has again been demonstrated in the context of our patient, where it facilitated safe and well anaesthetised videolaryngoscopy- sparing the need for fibreoptic methods.

Intubating in the absence of muscle paralysis contributes a risk of laryngospasm [15]. Given previously demonstrated difficulty of bag mask ventilation in patient with TCS [4], and therefore the likely difficulty in providing positive end expiratory pressure (PEEP), laryngospasm was identified as a critical concern. The airway plan (Figure 3 ) considers at the point of identifying a favourable view, either direct vocal cord topicalisation or muscle relaxation. The anaesthetic team were confident in this situation that thorough topicalisation was possible. Together with an appropriate depth of propofol-induced anaesthesia and CoPhenylcaine spray to the oropharnyx, we believe we provided the safest possible conditions toward avoiding larnygospasm without requiring muscle paralysis. Admittedly, given the favourability of the glottic view it is likely that the degree in overall risk between our technique and adminstering rocuronium was rather negligible. The intubating conditions would have likely only improved with muscle relaxation and Sugammadex $(16 \mathrm{mg} / \mathrm{kg})$ was immediately available in theatre.

\section{Conclusion}

This case study seeks to build upon the sparse body of literature for airway management of adult Treacher Collins Syndrome (TCS) patients undergoing general anaesthesia. It demonstrates a case of successful intubation with hyperangulated videolaryngoscopy. Pivotal to the safety of this procedure was maintaining spontaneous respiration whilst providing an adequate depth of anaesthesia prior to airway instrumentation. This was achieved with STRIVE Hi, which safely maintained oxygenation and ventilation for our patient until the airway had been secured.

\section{References}

1. Raj D, Luginbuehl I. Managing the difficult airway in the syndromic child. Continuing Education in Anaesthesia Critical Care \& Pain. 2014; 15:7-13.

2. Marres HAM, Cremers CWRJ, Dixon MJ, Huygen PLM, Joosten FBM. The Treacher Collins syndrome: a clinical, radiological, and genetic linkage study on two pedigrees. Arch Otolaryngol Head Neck Surg. 1995;121(5):509-514.

3. Chang CC, Steinbacher DM. Treacher Collins Syndrome. Semin Plast Surg. 2012;26(2):83-90.

4. Ratnamma V, Navaratnarajah J. 141 Treacher collins syndrome - retrospective review of airway management techniques. Archives of Disease in Childhood. 2018;103:A56-A57.

5. Hosking J, Zoanetti D, Carlyle A, Anderson P, Costi D. Anesthesia for Treacher Collins syndrome: a review of airway management in 240 pediatric cases. Paediatr Anaesth. 2012;22:752-758.

6. Trainor P, Dixon J, Dixon M. Treacher Collins syndrome: etiology, pathogenesis and prevention. Eur JHum Genet. 2009; 17:275-283.

7. Inagawa G, Miwa T, Hiroki K. The Change of Difficult Intubation with Growth in a Patient with Treacher Collins Syndrome. Anesthesia \& Analgesia. 2004;99(6):1874.
8. Paloma M, Fornet I, De Miguel I, Palacio F, Lopez M. Airway Management in a Patient With Treacher Collins Syndrome Requiring Emergent Cesarean Section. Anesthesia \& Analgesia. 2007;105(1):294

9. Soh J, Hye WS, Sung UC, Choon HL, Hye WL. Easy airway management using the i-gel ${ }^{\text {Tw }}$ supraglottic airway in a patient with Treacher Collins syndrome. Korean J Anesthesiol. 2014;67:17-18.

10. Roca O, Hernandez G, Diaz-Lobato S, Carratala JM, Gutierrez RM, Masclans JR. Current evidence for the effectiveness of heated and humidified high flow nasal cannula supportive therapy in adult patients with respiratory failure. Crit Care. 2016;20:109

11. Nishimura M. High-flow nasal cannula oxygen therapy in adults: physiological benefits, indication, clinical benefits, and adverse effects. Respir Care. 2016;61:529-41

12. Diab S, Fraser JF. Maintaining oxygenation successfully with high flow nasal cannula during diagnostic bronchoscopy on a postoperative lung transplant patient in the intensive care. Case Rep Crit Care. 2014;2014:3

13. Booth AW, Vidhani K. Spontaneous ventilation using Propofol TCI for microlaryngoscopy in adults: a retrospective audit. Anaesth Intensive Care. 2016;44(2):285-93 
14. Booth AWG, Vidhani K, Lee PK, Thomsett CM. SponTaneous Respiration using IntraVEnous anaesthesia and Hi-flow nasal oxygen (STRIVE Hi) maintains oxygenation and airway patency during management of the obstructed airway: an observational study. $\mathrm{Br} J$ Anaesth. 2017;118:444451

15. Lee PK, Booth AWG, Vidhani K. Spontaneous Respiration Using Intravenous Anesthesia and High-Flow Nasal Oxygen (STRIVE Hi) Management of Acute Adult Epiglottitis: A Case Report.AAPract. 2018;10(4):73-75
16. Verathon. Washington USA. GLIDESCOPE SPECTRUM SINGLE-USE [cited 2020 Apr 21]. Availablefrom: https://www.verathon.com/glidescope-spectrum-single-use/

17. (Editor) Riley R. Australia New Zealand College of Anaesthetists [Internet]. Western Australia: ANZCA; 2019 [cited 2020 Apr 21].3-46. Available from http://www.anzca.edu.au/documents/blue-book-2019.pdf
Conflict of Interest: Nil

Source of Support: None

\section{How to Cite this Article}

Young L, Potgieter D W, Roche C | Intubating a Treacher Collins Syndrome patient with STRIVE Hi and videolaryngoscopy | Journal of Anaesthesia and Critical Care Case Reports | May-August 2020; 6(2): 06-09. 